\title{
ON REPRESENTATIONS AND EXTENSIONS OF BOUNDED LINEAR FUNCTIONALS DEFINED ON CLASSES OF ANALYTIC FUNCTIONS
}

\author{
BY \\ PHILIP DAVIS AND J. L. WALSH
}

1. Introduction. In treatments of the subject of interpolation and approximation for complex analytic functions, it is usual to deal with the theory of interpolation series and the theory of complex analytic Fourier (i.e., orthogonal) series separately. Interpolation series are generally associated with a sequence of point functionals such as $f\left(\alpha_{n}\right)$ or $f^{(n)}\left(\alpha_{n}\right)$, and many interpolation series possess "Taylor-like" convergence properties. On the other hand, complex analytic Fourier series are associated with a sequence of integral inner products, and the theory carries with it the usual least squares best approximation properties of expansions in orthogonal functions. From a purely formal point of view, however, and even in the real case, these two types of expansions have many properties in common. The principal structural feature of both types of expansions is their use of biorthogonal sets. That is to say, in both theories we are confronted with a set of functions $\left\{\phi_{n}(z)\right\}$ and a set of linear functionals $\left\{L_{n}\right\}(n=0,1, \ldots)$ which are biorthogonal in the sense that

$$
L_{m}\left(\phi_{n}\right)=\delta_{m n} \quad(m, n=0,1, \cdots)
$$

and we study the convergence and representability properties of the biorthogonal expansion of an arbitrary function

$$
f(z) \sim \sum_{n=0}^{\infty} L_{n}(f) \phi_{n}(z)
$$

The biorthogonal sets $\left\{L_{n}\right\}$ and $\left\{\phi_{n}\right\}$ may be obtained in several ways. There is firstly the method of Fourier series wherein a given linearly independent set $\left\{\psi_{n}\right\}$ is orthogonalized over some point set $S$ yielding

$$
\phi_{n}=\sum_{k=0}^{n} a_{n k} \psi_{k}
$$$$
(n=0,1, \cdots)
$$

and $(1)$

$$
L_{n}(f)=\int_{S} f \overline{\phi_{n}} d \mu
$$

Received by the editors December 31, 1952.

(1) $\phi^{-}$denotes the conjugate imaginary of $\phi ; B^{-}$denotes the closure of $B$. 
with

$$
\int_{S} \phi_{n} \overline{\phi_{m}} d \mu=\delta_{m n}
$$

There is secondly the method of interpolation series wherein a given set of functionals $\left\{l_{n}\right\}$ and a given set of functions $\left\{\psi_{n}\right\}$ are biorthogonalized yielding

$$
L_{n}(f)=\sum_{k=0}^{n} b_{n k} l_{k}, \quad \phi_{n}=\sum_{k=0}^{n} a_{n k} \psi_{k}
$$

for which (1) holds. There are also various mixed methods which yield doubly orthogonal sets of functions, orthogonality for each set of functions occurring twice: either twice in the sense of (1) or twice in the sense of (5), or once in the sense of (1) and once in the sense of $(5)\left({ }^{2}\right)$.

Whether the functionals $L_{n}$ are point functionals or integral inner products, the purely algebraic theories of biorthogonal expansions (2) are identical and lead to the formation of certain determinants of Gram type. In the real case, and ostensibly in the complex analytic case, point functionals are distinguishable from integral functionals in that they may be defined for a wider class of functions. Thus, e.g., the functional $L_{1}(f)=f(0)$ is applicable to all functions $f(x)$ which are defined at $x=0$, whereas $L_{2}(f)=\int_{-1}^{1} f(x) d x$ is defined only over the set of functions $L^{1}[-1,+1]$. If, however, we limit ourselves to the case of analytic functions, and deal with functionals which are bounded in some sense, then this difference is largely illusory, and, as we shall show, there is almost complete identity between the two types of functional. Any bounded linear functional may be expressed as an infinite series of appropriately selected point functionals and may be approximated uniformly by a finite selection of such functionals. For this reason, the theory of interpolation series and the theory of Fourier series are closely bound, and in a recent paper $\left({ }^{3}\right)$, the authors were able to exhibit nontrivial series expansions related to an arbitrary region which simultaneously possess all the properties associated with interpolation series and Taylor-like convergence, and all the properties associated with Fourier expansions. Inner products associated with such expansions and defined by integrals may frequently be extended to a wider class of functions, and we may therefore speak of "generalized Fourier coefficients" given by point functionals $\left({ }^{4}\right)$. To exhibit the property of the ex-

(2) Other methods of generating biorthogonal sets are also possible. Thus, e.g., J. M. Whittaker and his co-workers have studied expansions in so-called basic sets of polynomials. Starting from a given basic set of polynomials, we may, by the consideration of the inverse of its infinite coefficient matrix, introduce a biorthogonal set of linear differential operators of infinite order. Cf. J. M. Whittaker [10]. See also Widder [11].

(3) Walsh-Davis (9). Cf. also $\$ 3$.

(4) For some properties of these generalized coefficients, see Davis-Pollak [5]. 
tendability of functionals at its simplest, consider, say, the two formulas

$$
\begin{aligned}
& L_{1}(f)=(2 \pi i)^{-1} \int_{C} \frac{f(z)}{z-z_{0}} d z, \\
& L_{2}(f)=f\left(z_{0}+\epsilon_{0}\right)+\sum_{n=1}^{\infty}\left(f\left(z_{0}+\epsilon_{n}\right)-f\left(z_{0}+\epsilon_{n-1}\right)\right) ; \quad \epsilon_{n} \rightarrow 0 .
\end{aligned}
$$

If $f(z)$ is regular in and on the contour $C$ containing $z_{0}$ in its interior, then we shall have $L_{1}(f) \equiv L_{2}(f)$. Yet the two formulas on the right are operationally different. The formula (8) has a wider applicability than (7), and may be said to define an extension of the intrinsic functional of which both (7) and (8) are but functional elements. The analogy with the continuation of analytic functions is obvious.

It is therefore of considerable interest to study representation and extendability properties for bounded linear functionals defined over certain classes of analytic functions, and this is the object of the present paper. The classical representation theorem of F. Riesz on bounded linear functionals is of prime importance here, but inasmuch as we shall not be dealing with a fixed Hilbert space of analytic functions, we cannot refer all properties of functionals to those of functions by this device. Indeed, we shall be concerned also with the Taylor aspects of functionals and with a border line region which relates the Taylor and the Fourier aspects. It should be pointed out moreover that in dealing with linear functionals as such, we are close to the theory of the calculus of symbolic operators $\left({ }^{5}\right)$. At the outset we shall mention some general results for Hilbert spaces of analytic functions.

2. Some general theorems. Let $B$ be a region lying in the complex $z=x+i y$ plane. By $H(B)$ we designate a set of functions which are singlevalued and analytic over $B$ and which form a Hilbert space with an inner product $(f, g)$ and a norm $\|f\|=(f, f)^{1 / 2}$. We shall show that the simplest point functionals of interpolation theory are bounded functionals over $H(B)$ provided that $H(B)$ possesses a reproducing kernel. A function of two complex variables $K\left(z_{1}, z_{2}\right)$ is called a reproducing $\operatorname{kernel}\left({ }^{6}\right)$ for $H(B)$ if $K$ is defined for $\left(z_{1}, z_{2}\right) \in B \times B$, if for each fixed $z_{2} \in B, K\left(z_{1}, z_{2}\right) \in H(B)$ as a function of $z_{1}$ and if the characteristic reproducing property

$$
f\left(z_{2}\right)=\left(f\left(z_{1}\right), K\left(z_{1}, z_{2}\right)\right)
$$

holds for all $z_{2} \in B$ and all $f \in H(B)$. In (9), the inner product operation is understood as applying to the variable $z_{1}$. On the basis of these properties, it is readily shown that if a reproducing kernel exists, it is unique, is Hermitian symmetric; and we shall emphasize the fact by writing $K\left(z_{1}, z_{2}^{-}\right)$instead of $K\left(z_{1}, z_{2}\right)$. We prove, now, a necessary and sufficient condition that the functional

(5) See, e.g., Pincherle [7].

(6) Bergman [2]; Aronszajn [1] contains an extensive abstract treatment of this subject. 


$$
L(f)=f\left(z^{*}\right)
$$

be bounded over $H(B)$ for each $z^{*} \in B$ is that $H(B)$ possess a reproducing kernel $K\left(z_{1}, z_{2}\right)$. Moreover, the possession of a kernel which is bounded on every closed bounded subset of $B$ is sufficient to guarantee that the functionals

$$
L_{n}(f)=f^{(n)}\left(z^{*}\right) \quad(n=0,1, \cdots)
$$

are each bounded over $H(B)$. Suppose, first, that $H(B)$ possesses a reproducing kernel. Then $L(f)=f\left(z^{*}\right)=\left(f\left(z_{1}\right), K\left(z_{1}, z^{*-}\right)\right)$, and therefore by the Schwarz inequality, $|L(f)|^{2} \leqq\|f\|^{2}\left(K\left(z_{1}, z^{*-}\right), K\left(z_{1}, z^{*-}\right)\right) \leqq\|f\|^{2} K\left(z^{*}, z^{*-}\right)$, proving the boundedness of $L$. Conversely, if $L$ is bounded, then by a classic theorem of $F$. Riesz, there exists for each $z^{*} \in B$ a function $g\left(z, z^{*}\right) \in H(B)$ such that $L(f)$ $=f\left(z^{*}\right)=\left(f\left(z_{1}\right), g\left(z_{1}, z^{*}\right)\right)$. Thus, $g\left(z, z^{*}\right)$ serves as a reproducing kernel. As regards the second statement, let $\left\{\phi_{n}(z)\right\}$ be a complete orthonormal system for $H(B)$. For a given $f \in H(B)$, its Fourier series $f(z) \sim \sum_{n=0}^{\infty} \alpha_{n} \phi_{n}(z), \alpha_{n}=\left(f, \phi_{n}\right)$, converges to $f$ in norm. Now, $\left|f(z)-\sum_{n=0}^{N} \alpha_{n} \phi_{n}(z)\right| \leqq\left\|f-\sum_{n=0}^{N} \alpha_{n} \phi_{n}\right\|$ - $\left[K\left(z, z^{-}\right)\right]^{1 / 2}$, so that if $z$ is confined to a closed bounded subset of $B$, the Fourier series for $f$ will converge to $f$ pointwise and uniformly. Thus, if $C$ designates a simple closed curve lying in $B$ and containing $z^{*}$ in its interior we have

$$
L_{n}(f)=\frac{n !}{2 \pi i} \int_{C} \frac{1}{\left(z-z^{*}\right)^{n+1}} \sum_{k=0}^{\infty} \alpha_{k} \phi_{k}(z) d z=\sum_{k=0}^{\infty} \alpha_{k} L_{n}\left(\phi_{k}\right) .
$$

The last member of (12) converges for all $\sum_{k=0}^{\infty}\left|\alpha_{k}\right|^{2}<\infty$, and by a result of Landau, this implies that $\sum_{k=0}^{\infty}\left|L_{n}\left(\phi_{k}\right)\right|^{2}<\infty$. Thus, finally, $\left|L_{n}(f)\right|^{2}$ $\leqq \sum_{k=0}^{\infty}\left|\alpha_{k}\right|^{2} \sum_{k=0}^{\infty}\left|L_{n}\left(\phi_{k}\right)\right|^{2}$, establishing the result. The same result obviously holds where $L$ is any one of a wide class of integral operators taken over a closed bounded point set lying in $B$. Extensions to linear differential operators of infinite order may also be made in this way.

Let $H(B)$ and $H(G)$ designate two Hilbert spaces of functions which are analytic in regions $B$ and $G$, respectively. The Hilbert spaces possess inner products $(f, g)_{B}$ and $(f, g)_{G}$, and we shall suppose that $H(B) \subset H(G)$ in the following sense

$$
\begin{gathered}
G \subset B, \\
\text { if } f \in H(B) \text { then } f \in H(G) .
\end{gathered}
$$

If, in addition, we have for all $f \in H(B)$,

$$
\|f\|_{B} \geqq\|f\|_{G},
$$

we shall speak of the space $H(B)$ as possessing a subnorm property with respect to $H(G)$. One consequence of the subnorm property is that a linear functional which is bounded over $H(G)$ is also bounded over $H(B)$. A second 
consequence $\left.{ }^{7}\right)$ is that if $H(G)$ possesses a reproducing kernel, then $H(B)$ possesses a reproducing kernel $K_{H(B)}\left(z_{1}, z_{2}\right)$.

If $H(B)$ possesses the reproducing kernel $K\left(z_{1}, z_{2}^{-}\right)$, and if $L$ designates a bounded linear functional over $H(B)$, then the Riesz representative $l(z)$ of $L$ may be expressed compactly in terms of $K$. Indeed, we have $l\left(z_{2}\right)$ $=\left(L_{z_{1}} K\left(z_{1}, z_{2}^{-}\right)\right)-$where the bar indicates the conjugate imaginary. To show this, consider $\left(L_{z_{1}} K\left(z_{1}, z_{2}^{-}\right)\right)^{-}=\left(\left(K\left(z_{1}, z_{2}^{-}\right), l\left(z_{1}\right)\right)_{z_{1}}\right)^{-}=\left(l\left(z_{1}\right), K\left(z_{1}, z_{2}^{-}\right)\right)_{z_{1}}=l\left(z_{2}\right)$. The importance of this representation arises when $L$ is given initially by a rule other than that of inner product in $H(B)$.

Let $L$ designate a linear functional which is bounded over $H(B)$. We shall say that $L$ is extendable to $H(G)$ if there exists a linear functional $L^{*}$ which is bounded over $H(G)$ and such that $L^{*}$ coincides with $L$ on $H(B)$, i.e., $L^{*}(f)$ $=L(f)$ for all $f \in H(B)$. In general, given a linear functional $L$ which is bounded over $H(B)$, it will not be possible to effect an extension of $L$ to $H(G)$ (cf. §3). This is true even when $H(B)$ is dense in $H(G)$; there is, however, no conflict here with the Hahn-Banach extension theorem inasmuch as the linear functional $L$ though defined and bounded on $H(B)$ in the sense of the norm \|\|$_{B}$ may not be bounded in the sense of the norm \|\|$_{G}$.

Assuming that $L$ is bounded over $H(B)$ and can be extended to $H(G)$ yielding an $L^{*}$, this extension will be unique provided that $H(B)$ is dense in $H(G)$. For, suppose that a given $L$ has two extensions $L^{*}$ and $L^{* *}$ which are bounded over $H(G)$. We construct the linear functional $L^{\prime}=L^{*}-L^{* *}$ which is bounded over $H(G)$, and which vanishes identically over $H(B)$. Now let $g \in H(G)$. For a given $\epsilon>0$, we may find an $f \in H(B)$ such that $\|g-f\|_{G}<\epsilon$. Thus $\left|L^{\prime}(g-f)\right|=\left|L^{\prime}(g)\right| \leqq M \epsilon$, and thus $L^{\prime}$ must vanish over $H(G)$. Conversely, suppose that $H(B)$ is not dense in $H(G)$. Then there exist linear functionals $L^{\prime}$ which vanish identically over $H(B)$, do not vanish identically over $H(G)$, and are bounded over $H(G)$. For let $\left\{\phi_{n}(z)\right\}$ be a complete orthonormal set for $H(B)$. By hypothesis, this set cannot be complete for $H(G)$, but will become complete by the adjunction of a finite or at most denumerable number of functions $\psi_{n}(z) \in H(G)$. Each $\psi_{n}$ may be selected moreover so as to be orthogonal to the subspace $H(B)$. Let $\left\{\beta_{n}\right\}$ be an arbitrary sequence of constants for which $\sum_{n=0}^{\infty}\left|\beta_{n}\right|^{2}<\infty$, and define a linear functional $L^{\prime}$ as follows: if $g \in H(G)$ and possesses the Fourier expansion $g$ $=\sum_{n=0}^{\infty} \alpha_{n} \phi_{n}+\sum_{n=0}^{\infty} a_{n} \psi_{n}$, then $L^{\prime}(g)=\sum_{n=0}^{\infty} \beta_{n} a_{n} . L^{\prime}$ is now seen to possess the desired property, and this means that in such a case, any extension from $H(B)$ to $H(G)$ cannot be unique.

Let $L$ be a linear functional which is bounded over $H(B)$. Let $\left\{\phi_{n}(z)\right\}$ be a complete orthonormal system for $H(G)$. Then a necessary and sufficient condition that $L$ be extendable to $H(G)$ is that there exist a linear functional $L^{*}$ which has the following two properties: (a) $L(f)=L^{*}(f)$ for all $f$ in $H(B)$, and (b) $\sum_{n=0}^{\infty}\left|L^{*}\left(\phi_{n}\right)\right|^{2}<\infty$. The condition is necessary. For if there is an extension

(7) Aronszajn [1, p. 355]. 
$L^{*}$, then (a) obviously holds. Now for any $g \in H(G), L^{*}(g)=\left(g, g^{*}\right)_{G}$ for some $g^{*} \in H(G)$. Again $g$ has a Fourier series $\sum_{n=0}^{\infty} a_{n} \phi_{n}$ convergent in norm to $g$ if and only if $\sum_{n=0}^{\infty}\left|a_{n}\right|^{2}<\infty$. Thus $L^{*}(g)=\left(\sum_{n=0}^{\infty} a_{n} \phi_{n}, g^{*}\right)=\sum_{n=0}^{\infty} a_{n} L^{*}\left(\phi_{n}\right)$. Therefore, by Landau's theorem, $\sum_{n=0}^{\infty}\left|L^{*}\left(\phi_{n}\right)\right|^{2}<\infty$. Conversely, let there exist an $L^{*}$ with properties (a) and (b). If for any $g \in H(G), g \sim \sum_{n=0}^{\infty} a_{n} \phi_{n}$, $\sum_{n=0}^{\infty}\left|a_{n}\right|^{2}<\infty$, we define $L^{*}(g)$ by $L^{*}(g)=\sum_{n=0}^{\infty} a_{n} L^{*}\left(\phi_{n}\right)$, it is clear that $L$ possesses an extension which is bounded in the sense of $H(G)$.

As we shall see below, it will be possible to convert (a) and (b) into a criterion which can be applied directly to $L$.

We shall say that the set $\left\{L_{n}\right\}$ of functionals is complete for $H(B)$ if $L_{n}(f)$ $=0(n=0,1, \cdots), f \in H(B)$ implies that $f$ vanishes identically. If $f_{n}$ is the Riesz representative of $L_{n}$, then the completeness of $\left\{f_{n}\right\}$ as functions is necessary and sufficient for the completeness of the set $\left\{L_{n}\right\}$. As a particular case, we observe that if $B$ contains the point $z^{*}$ in its interior, then the sets $L_{n}(f) \equiv f^{(n)}\left(z^{*}\right) \quad(n=0,1, \cdots) ; L_{n}(f)=f\left(\alpha_{n}\right), \lim _{n \rightarrow \infty} \alpha_{n}=z^{*}$, are complete. These sets will be complete for all spaces $H(B)$, and in special cases, e.g., if $H(B)$ contains only entire functions of limited exponential type, other "less stringent" sets may replace them. The importance of complete sets of functionals for the theory of interpolation lies in the fact that (as in the case of functions) any bounded linear functional may be expanded in a series of linear combinations of functionals of such a set, hence in particular may be expanded in terms of any complete set of bounded linear interpolatory functionals. Indeed, let $\left\{L_{n}\right\}$ be a complete set and $L$ a given functional while $\left\{g_{n}\right\}$ and $g$ are the respective Riesz representatives. The set of functions $\left\{g_{n}\right\}$ is complete and may be orthonormalized yielding $g_{n}^{*}=\sum_{k=0}^{n} a_{n k} g_{k}$. We now have a Fourier expansion $g \sim \sum_{n=0}^{\infty} \alpha_{n} g_{n}^{*}$, convergent in norm, and hence, transforming again to the functional space,

$$
L=\sum_{n=0}^{\infty} \alpha_{n} \sum_{k=0}^{n} a_{n k} L_{k} .
$$

Convergence to $L(f)$ of this series when applied to any $f \in H(B)$ is readily established. The expansion (13) is equivalent to starting with a biorthogonal expansion $f(z) \sim \sum_{n=0}^{\infty} L_{n}^{*}(f) g_{n}^{*}(z)$, applying $L$ to both sides formally, and arriving at

$$
L=\sum_{n=0}^{\infty} L\left(g_{n}^{*}\right) L_{n}^{*}, \quad\|L\|^{2}=\sum_{n=0}^{\infty}\left|L\left(g_{n}^{*}\right)\right|^{2},
$$

as an orthogonal expansion for the functional $L$. As particular cases we may select $L_{n}(f)=f^{(n)}(0)$, or $L_{n}(f)=f\left(\alpha_{n}\right), \lim _{n \rightarrow \infty} \alpha_{n}=0$, leading to

$$
L(f)=\sum_{n=0}^{\infty} \alpha_{n} \sum_{k=0}^{n} a_{n k} f^{(k)}(0)\left({ }^{8}\right),
$$

(8) Cf. Davis [4]. 


$$
L(f)=\sum_{n=0}^{\infty} \alpha_{n} \sum_{k=0}^{n} a_{n k}^{\prime} f\left(\alpha_{k}\right) .
$$

The expansions (15) and (16) may be written in the form

$$
\begin{aligned}
& L(f)=\frac{1}{2 \pi i} \sum_{n=0}^{\infty} \alpha_{n} \int_{C} R_{n}^{(1)}(z) f(z) d z, \\
& L(f)=\frac{1}{2 \pi i} \sum_{n=0}^{\infty} \alpha_{n} \int_{C} R_{n}^{(2)}(z) f(z) d z
\end{aligned}
$$

with

$$
\begin{aligned}
& R_{n}^{(1)}(z)=\sum_{k=0}^{n} a_{n k} \frac{k !}{z^{k+1}}, \\
& R_{n}^{(2)}(z)=\sum_{k=0}^{n} \frac{a_{n k}^{\prime}}{z-a_{k}},
\end{aligned}
$$

and $C$ designates a simple closed curve lying in $B$ containing 0 or the points $a_{k}$ in its interior. The sets of rational functions $R^{(i)}(z)$ depend solely upon the domain $B$ and upon the inner product of the space $H(B)$, but are independent of the particular functional to be expanded. If the order of summation and integration can be reversed in (17) or (18), then there is obtained a representation of $L$ as a Cauchy integral. As we shall see subsequently, this is not always possible, and the whole question is intimately related to the analytic continuability of the Riesz representative of $L$. A further consequence of the expansions (15) and (16) is that every bounded linear functional over $H(B)$ is uniformly approximable by a finite number of point or differential operators.

Let $H(B)$ be a Hilbert space of analytic functions defined on a region $B$ containing the origin in its interior and possessing $K_{H(B)}$ and an inner product $(f, g)$. Let $\left\{g_{n}\right\}$ be a complete orthonormal system and introduce the sequence of bounded functionals $L_{n}(f)=\left(f, g_{n}\right)$. We have now for appropriate constants $a_{n k}^{(p)}, L_{p}(f)=\sum_{n=0}^{\infty} \sum_{k=0}^{n} a_{n k}^{(p)} f^{(k)}(0)$. Hence, a necessary and sufficient condition that any function $f(z)$ regular in a neighborhood of the origin belong to $H(B)$ is that $\sum_{p=0}^{\infty}\left|\sum_{n=0}^{\infty} \sum_{k=0}^{n} a_{n k}^{(p)} f^{(k)}(0)\right|^{2}<\infty$. This condition is also necessary and sufficient that $f(z)$ in $H(B)$ should exist when merely the numbers $f^{(k)}(0)$ are given. It is to be observed that this criterion may be applied to all $f$ which are regular at 0 , whereas the inner product rule $(f, g)$ may be much more limited in its applicability. Such a situation arises, e.g., where the inner product is a line integral taken over the boundary of the domain of analyticity.

3. Extensions for the class $L^{2}(B)$. We turn now to some concrete representations of the situations outlined in the previous section. Let $B$ designate a bounded region. By $L^{2}(B)$, we designate the space of functions which are 
single-valued and analytic in $B$ and possess finite norm $\|f\|_{B}^{2}=\iint_{B}|f|^{2} d x d y\left({ }^{9}\right)$. $L^{2}(B)$ is a Hilbert space with an inner product $(f, g)_{B}=\iint_{B} f g^{-} d x d y$, and a hermitian symmetric reproducing kernel $K_{B}\left(z, w^{-}\right)$. For another region $G \subset B$, we have $L^{2}(B) \subset L^{2}(G)$, and the space $L^{2}(B)$ has the subnorm property $\|f\|_{B} \geqq\|f\|_{G}$ for all $f \in L^{2}(B)$. If $L$ is bounded over $L^{2}(B)$, its norm is given by $\|L\|^{2}=L_{z} L_{w} K_{B}\left(z, w^{-}\right)$. For a point $z^{*} \in B$, each functional $L(f)$ $=f^{(n)}\left(z^{*}\right)(n=0,1, \cdots)$ is bounded.

Certain converses of this statement can be established. Let $B$ be a bounded region and let $G$ be simply connected, possess a Jordan boundary, and $G^{-} \subset B$. If $z^{*}$ is in $B$ but is exterior to $G$, the functional $L(f)=f^{(j)}\left(z^{*}\right)$ for fixed $j=0,1, \ldots$ cannot be extended so as to be bounded over $L^{2}(G)$. If $G$ possesses an analytic Jordan boundary, the same statement holds when $z^{*}$ lies on its boundary.

To show this we need only find a sequence of functions $r_{n}(z)$, each of class $L^{2}(B)$ and such that

$$
\limsup _{n \rightarrow \infty}\left|r_{n}^{(j)}\left(z^{*}\right)\right| /\left\|r_{n}\right\|_{G}=\infty,
$$

for fixed $j=0,1, \cdots$. If $z^{*}$ is exterior to $G$ we may find an analytic curve $C$ containing $G^{-}$in its interior and with $z^{*}$ lying exterior to $C$. Thus, e.g., we may take a kreisbild of the conformal map of the exterior of $G$ onto the exterior of a circle. If the boundary of $G$ is itself analytic, and if $z^{*}$ lies on it, we take $C=$ the boundary of $G$. Let $\left\{p_{n}(z)\right\}$ designate the set of polynomials which are orthonormal over the finite simply-connected region bounded by $C$. We shall show that $p_{n}$ satisfy (21). We indicate the proof for $j=0$. The details for the cases $j=1,2, \cdots$ are then readily supplied.

The polynomials $p_{n}$ have the following asymptotic expression( $\left.{ }^{10}\right)$ holding on and exterior to $C$ :

$$
p_{n}\left(z^{*}\right)=\left[\frac{n+1}{\pi}\right]^{1 / 2} \phi^{\prime}\left(z^{*}\right)\left[\phi\left(z^{*}\right)\right]^{n}+O\left(r^{n}\right)
$$

for some $0<r<1$, and where $\phi(z)$ designates the conformal map of the exterior of $C$ onto the exterior of the unit circle. Thus, we have

$$
\lim _{n \rightarrow \infty}\left|p_{n}\left(z^{*}\right)\right|=\infty \text {. }
$$

Now, finally, $\left\|p_{n}\right\|_{G} \leqq\left\|p_{n}\right\|_{\text {Int } C}=1(n=0,1, \cdots)$ so that $(21)$ follows for the sequence $p_{n}$.

If $L$ possesses an extension $L^{*}$ to $L^{2}(G)$, then the uniqueness of $L^{*}$ is, as we have seen, related to the denseness of $L^{2}(B)$ in $L^{2}(G)$. This in turn may be related to the geometry of the regions in question. Let, e.g., $B$ be the unit circle $|z|<1$ and $G$ be an annulus $A: 0<a<|z|<b<1$. Let $C$ designate a

(9) Cf. Bergman [2].

(10) Carleman [3], Korovkin [6]. 
simple closed curve lying interior to $A$ which is not reducible to a point in $A$. The functional $L^{*}(f)=\int_{c} f(z) d z$ is 0 for $f \in L^{2}(B)$, but is not identically zero over $L^{2}(G)$. No extension to $L^{2}(G)$ is unique. Counter-examples may be found even when $G$ is simply connected. A sufficient condition for the uniqueness of extensions is that $G$ be simply connected, and possess a boundary $g$ such that the points of the plane which do not lie in $G+g$ form a single region whose boundary is precisely $g$. In this case, the set of powers of $z$ is complete over $L^{2}(G)$ and a fortiori $L^{2}(B)$ is dense in $L^{2}(G)$.

We shall say that a functional $L$ which is bounded over $L^{2}(B)$ possesses a Cauchy representative if there exists a simple closed rectifiable curve $C$ contained in the interior of $B$ and an integrable complex-valued function $c(z)$ defined on $C$ such that for all $f \in L^{2}(B)$, we have

$$
L(f)=\int_{C} c(z) f(z) d z .
$$

The first observation that should be made is that if $L$ possesses a Cauchy representative, then it possesses an analytic Cauchy representative. For, let

$$
c^{*}(z)=\frac{1}{2 \pi i} \int_{c} \frac{c(w)}{z-w} d w .
$$

$c^{*}(z)$ is clearly analytic in the exterior of $C$, and is $O(1 / z), z \rightarrow \infty$. Let $C^{\prime}$ be a simple closed rectifiable curve in $B$ which contains $C$ in its interior and which is homotopic to $C$. Then

$$
\begin{aligned}
\int_{C^{\prime}} c^{*}(z) f(z) d z & =\frac{1}{2 \pi i} \int_{c^{\prime}} f(z) d z \int_{c} \frac{c(w)}{w-z} d w \\
& =\frac{1}{2 \pi i} \int_{C} c(w) d w \int_{C^{\prime}} \frac{f(z)}{z-w} d z \\
& =\int_{c} c(w) f(w)=L(f) .
\end{aligned}
$$

An analytic Cauchy representative $c(z)$, if it exists, will not be unique, for we may obviously add to $c(z)$ any function which is regular in and on $C$. But it is easily proved unique if it is required, in addition, that it be regular exterior to $C$ and vanish at infinity.

$A$ linear functional $L$ which is bounded over $L^{2}(B)$ possesses a Cauchy representative if and only if $L$ may be extended to some $L^{2}(G)$ with $G-C B$. For if $L$ has a Cauchy representative, then (24) gives an obvious extension to $L^{2}(G)$ where $G$ is any region containing $C$ in its interior. The functional defined by (24) is bounded over $L^{2}(G)$. For, $|L(f)| \leqq M l(C) \max _{z} \in C|f(z)|$, where $M=\max _{z \in C}|c(z)|$ and $l(C)=$ length of $C$. Now $|f(z)| \leqq\left\{K_{G}(z, z)\right\}^{1 / 2}\|f\|_{G}$, so that boundedness over $L^{2}(G)$ follows. Conversely, let $G^{-} C B$ and let $L$ 
be bounded over $L^{2}(G) . L$ is also bounded over $L^{2}(B)$, and we wish to show that $L$ (over $L^{2}(B)$ ) possesses a Cauchy representative. Let $l(z)$ be the Riesz representative of $L$ over $L^{2}(G)$ and define

$$
c(z)=\frac{1}{2 \pi i} \iint_{G} \frac{[l(w)]}{z-w} d A_{w} .
$$

The function $c(z)$ is obviously regular exterior to $G$. Let $C$ be a simple closed rectifiable curve lying in $B$, containing $G$ in its interior, and which can be deformed continuously to the boundary of $G$ within $B$. (For simplicity of exposition we suppose $G$ simply connected.) Then, for $f(z) \in L^{2}(B)$,

$$
\begin{aligned}
\int_{C} c(z) f(z) d z & =\frac{1}{2 \pi i} \int_{C} f(z) d z \iint_{G} \frac{[l(w)]^{-}}{z-w} d A_{w} \\
& =\iint_{G}[l(w)]-d A_{w} \frac{1}{2 \pi i} \int_{C} \frac{f(z)}{z-w} \alpha d z \\
& =\iint_{G} f(w)[l(w)]-d A_{w}=L(f) .
\end{aligned}
$$

If a functional $L$ possesses a Cauchy representative $c(z)$, then as has been already observed, its Riesz representative is given by $\left(L_{z} K_{B}(z, w)\right)^{-}$or,

$$
[l(z)]^{-}=\int_{C} c(w) K_{B}\left(z^{-}, w\right) d w .
$$

Conversely, let $L$ be bounded over $L^{2}(B)$, and possess Riesz representative $l(z)$. If

$$
c(z)=\frac{1}{2 \pi i} \iint_{B} \frac{[l(w)]-}{z-w} d A_{w}
$$

is regular in the exterior of a region $G$ for which $G^{-} \subset B$, and if, moreover, $B$ is such that there exists a complete orthonormal system of functions for $B$ which are regular in a region $B^{+}: B^{-} C B^{+}$, then $c(z)$ is the Cauchy representative of $L$.

The above hypotheses on $B$ will be satisfied in most instances of interest. Formula (30) may be written in the symbolic form

$$
c(z)=\frac{1}{2 \pi i} L_{w}\left(\frac{1}{z-w}\right) .
$$

To prove this statement, let $C$ be a simple closed curve lying in $B$ and in the region of regularity of $c(z)$ and set

$$
L^{*}(f)=\int_{c} c(z) f(z) d z ; \quad f \in L^{2}(B) .
$$


Let $\left\{\phi_{n}(z)\right\}$ be a complete system for $L^{2}(B)$ each function of which is regular in $B^{+}$. We have

$$
L^{*}\left(\phi_{n}\right)=\int_{C} c(z) \phi_{n}(z) d z=\int_{C^{*}} c(z) \phi_{n}(z) d z
$$

where $C^{*}$ is a simple closed curve which contains $G$ in its interior, and is itself contained in the interior of $B^{+}$. Now,

$$
\begin{aligned}
\int_{C^{*}} c(z) \phi_{n}(z) d z & =\frac{1}{2 \pi i} \int_{C^{*}} \phi_{n}(z) d z \iint_{B} \frac{[l(w)]-}{z-w} d A_{w} \\
& =\iint_{B}[l(w)]-d A_{w} \frac{1}{2 \pi i} \int_{C^{*}} \phi_{n}(z) \frac{1}{z-w} d z \\
& =\iint_{B}[l(w)]-\phi_{n}(w) d A_{w}=L\left(\phi_{n}\right) .
\end{aligned}
$$

Finally, for an arbitrary $f \in L^{2}(B)$, let $\left\|f-\sum_{n=0}^{N} \quad a_{n} \phi_{n}\right\|<\epsilon$. We have $\left|L^{*}(f)-L(f)\right|=\left|L^{*}(f)-\sum_{n=0}^{N} \quad a_{n} L^{*}\left(\phi_{n}\right)+\sum_{n=0}^{N} \quad a_{n} L\left(\phi_{n}\right)-L(f)\right| \leqq\left(\left\|L^{*}\right\|\right.$ $+\|L\|) \cdot\left\|f-\sum_{n=0}^{N} a_{n} \phi_{n}\right\|=o(1), N \rightarrow \infty$.

If $B$ is the unit circle, the relation between the Riesz representative $l(z)$ and the Cauchy representative $c(z)$ is given also by

$$
c(z)=\frac{1}{2 i} R^{-}\left(\frac{1}{z}\right) ; \quad R(z)=\int_{0}^{z} l(z) d z .
$$

This may be easily verified by a formal computation. If the orthogonalization is carried out not over the interior of the unit circle but over its circumference, leading to the space $H^{2}\left({ }^{11}\right)$, the relationship is even simpler:

$$
c(z)=\frac{1}{z} l-\left(\frac{1}{z}\right) .
$$

In both cases, suitable interpretation of the integral (24) can be introduced when $l(z)$ is an arbitrary function of the respective spaces.

In what follows, we shall utilize certain sets of orthogonal functions whose convergence properties were studied extensively by the authors in [9]. Here we assume that the boundary of $B$ consists of a finite number of mutually disjoint Jordan curves. Let $L_{n}(f)(n=0,1, \cdots)$ designate either (a) the set of linear functionals $f^{(n)}(0), 0 \in B$, or (b) $f\left(\alpha_{n}\right), \lim _{n \rightarrow \infty} \alpha_{n}=0, \alpha_{n} \in B$. Let $\phi_{n}(z)$ designate their Riesz representatives and suppose that the set $\left\{\phi_{n}(z)\right\}$ has been orthogonalized(12) over $B$ yielding $\left\{\phi_{n}^{*}(z)\right\}$. Let $L_{n}^{*}$ desig-

(11) See, e.g., Walsh [8, p. 141].

(12) In case (a) Bergman defines $\phi_{n}^{*}(z)$ as a suitable constant multiple of the function $\psi_{n}(z)$ with $\psi_{n}(0)=\psi_{n}^{\prime}(0)=\cdots=\psi_{n}^{(n-1)}(0)=0, \psi_{n}^{(n)}(0)=1$ of $L^{2}(B)$ whose norm is least. It is 
nate the set of functionals which are biorthogonal to $\phi_{n}^{*}$. Let $G(z, 0)$ designate the Green's function of $B$ with pole at $z=0$, and $C_{r}$ the set of level lines $G(z, 0)=-\log r$. The set $\left\{\phi_{n}{ }^{*}(z)\right\}$ possesses the following property. If $f(z)$ is regular in a neighborhood of $z=0$ and defined at $z=\alpha_{i}$, then the series

$$
f(z)=\sum_{n=0}^{\infty} L_{n}^{*}(f) \phi_{n}^{*}(z)
$$

will converge to $f(z)$ in the region interior to the largest $C_{r}$ in which $f$ is regular, uniformly on any closed set contained therein. If $f(z)$ is regular throughout $C_{r}, 0<r<1$, but throughout no region $C_{r^{\prime}}, r<r^{\prime} \leqq 1$, then we shall have

$$
\limsup _{n \rightarrow \infty}\left|L_{n}^{*}(f)\right|^{1 / n}=1 / r \text {. }
$$

The functionals $L_{n}^{*}$ are appropriate linear combinations of the $L_{k}$, and in case (a) we may write $L_{n}^{*}(f)=\sum_{k=0}^{n} a_{n k} f^{(k)}(0)$, while in case (b) we have $L_{n}^{*}(f)=\sum_{k=0}^{n} b_{n k} f\left(\alpha_{k}\right)$. For the purposes of the proof of the theorem which follows, we shall require a more precise form of the equation (38). Let $f(z)$ be analytic in and on $C_{r}, 0<r<1$. Then to every $r$ and to every $\epsilon>0$, there exists an $M=M(r, \epsilon)$, but which is independent of $f(z)$ and $n$, such that we have

$$
\left|L_{n}^{*}(f)\right| \leqq M\left(\int_{C_{r}}|f(z)|^{2} d s\right)^{1 / 2}\left(\frac{1}{r}+\epsilon\right)^{n} \quad(n=0,1, \cdots) .
$$

The inequality (39) was not derived in reference [9], but may be obtained by employing the techniques of that paper, and using known contour integral expressions for the coefficients of series expansions in Walsh rationals which were utilized in [9] as a set of auxiliary functions $\left({ }^{13}\right)$. We shall not pursue this matter further at the present time.

Theorem. Let $T$ be a linear functional which is bounded over $L^{2}(B)$ and which possesses the Riesz representative $t(z)$. If

$$
\limsup \left|T\left(\phi_{n}^{*}\right)\right|^{1 / n}=r \quad 0<r<1,
$$

or, what is equivalent,

readily proved by elementary means directly from this definition that a necessary and suffcient condition that a function $\psi(z)$ of $L^{2}(B)$ with $\psi(0)=\psi^{\prime}(0)=\cdots=\psi^{(n-1)}(0)=0$ be orthogonal to $\psi_{n}(z)$ is $\psi^{(n)}(0)=0$ (if either conclusion is not true, a suitable linear combination of $\psi(z)$ and $\psi_{n}(z)$ satisfies the auxiliary conditions on $\psi_{n}(z)$ and has a smaller norm). It follows at once that the formal expansion $\sum a_{n} \phi_{n}^{*}(z)$ of $f(z)$ of $L^{2}(B)$ found by interpolation in the origin is identical with the formal Fourier expansion of $f(z)$ in terms of the $\phi_{n}^{*}(z)$. A similar remark applies to case (b), using interpolation in the points $\alpha_{n}$, assumed to lie in $B$, even without the hypothesis $\alpha_{n} \rightarrow 0$.

(13) Walsh $[8$, p. 190.] 


$$
\underset{n \rightarrow \infty}{\limsup }\left|L_{n}^{*}(t(z))\right|^{1 / n}=r,
$$$$
0<r<1
$$

then $T$ may be extended so as to be bounded over $L^{2}\left(C_{r^{\prime}}\right), r^{\prime}>r$, but cannot be extended so as to be bounded over $L^{2}\left(C_{r^{\prime \prime}}\right), r^{\prime \prime}<r$. The converse is true and, moreover, appropriate modifications of statement may be made for the cases $r=1$, and $r=0$.

Proof. We shall prove first that if (40) holds, then $T$ may be extended so as to be bounded over $L^{2}\left(C_{r^{\prime}}\right), r^{\prime}>r$. Let $f(z) \in L^{2}\left(C_{r^{\prime}}\right)$. Then $f(z)$ possesses an expansion (37): $f(z)=\sum_{n=0}^{\infty} L_{n}^{*}(f) \phi_{n}^{*}(z)$, with $\lim \sup _{n \rightarrow \infty}\left|L_{n}^{*}(f)\right|^{1 / n} \leqq 1 / r^{\prime}$. Define a functional $T^{*}$ over $L^{2}\left(C_{r^{\prime}}\right)$ as follows:

$$
T^{*}(f)=\sum_{n=0}^{\infty} L_{n}^{*}(f) T\left(\phi_{n}^{*}\right) .
$$

From (40) and the last remark it appears that the series in (41) is dominated by $\sum_{n=0}^{\infty}\left(r+\epsilon_{1}\right)^{n}\left(1 / r^{\prime}+\epsilon_{2}\right)^{n}<\infty$, since $r / r^{\prime}<1$. Thus, (41) yields a legitimate definition for $T^{*}$, and in view of the fact that $\left\{\phi_{n}^{*}\right\}$ are orthonormal over $B$, $T^{*}(f)$ must coincide with $T(f)$ whenever $f \in L^{2}(B)$. It remains to show that $T^{*}$ is bounded over $L^{2}\left(C_{r^{\prime}}\right)$.

We introduce a complete set $\left\{q_{n}{ }^{*}(z)\right\}$ of orthonormal functions as follows: let $\left\{L_{n}\right\}$ be either (a) or (b) (cf. $\S$ preceding (37)) (14) and possess Riesz representatives $q_{n}(z)$ with respect to $L^{2}\left(C_{r^{\prime}}\right)$. Let $\left\{q_{n}(z)\right\}$ be orthonormalized by the Gram-Schmidt process over the interior of $C_{r^{\prime}}$ yielding $\left\{q_{n}^{*}(z)\right\}$ and the associated biorthogonal set of functionals $\left\{L_{n}^{* *}\right\}$. To prove the boundedness of $T^{*}$ over $L^{2}\left(C_{r^{\prime}}\right)$ we need only, by our remarks in $\$ 2$, show that

$$
\sum_{n=0}^{\infty}\left|T^{*}\left(q_{n}^{*}\right)\right|^{2}<\infty,
$$

or, by (41), show that

$$
\sigma^{2} \equiv \sum_{n=0}^{\infty}\left|\sum_{k=0}^{\infty} L_{k}^{*}\left(q_{n}^{*}\right) T\left(\phi_{n}^{*}\right)\right|^{2}<\infty .
$$

We now have

$$
L_{k}^{*}\left(q_{n}^{*}\right)=0 \quad(k=0,1, \cdots, n-1 ; n=0,1, \cdots),
$$

so that formally

$$
\sigma^{2}=\sum_{n=0}^{\infty}\left|\sum_{k=n}^{\infty} L_{k}^{*}\left(q_{n}^{*}\right) T\left(\phi_{n}^{*}\right)\right|^{2} \leqq \sum_{n=0}^{\infty}\left(\sum_{k=n}^{\infty}\left|L_{k}^{*}\left(q_{n}^{*}\right)\right|^{2}\right)\left(\sum_{k=n}^{\infty}\left|T\left(\phi_{n}^{*}\right)\right|^{2}\right) .
$$

From (40), we have $\left|T\left(\phi_{n}^{*}\right)\right|^{2} \leqq C_{1}(r+\epsilon)^{2 n}(n=0,1, \cdots)$, so that

(14) If the selection (b) is made, it will be assumed that all the points $z=\alpha_{n}$ lie interior to $C_{r^{\prime}}$. The omission of a finite number of these points may therefore be necessary. 


$$
\left(\sum_{k=n}^{\infty}\left|T\left(\phi_{n}^{*}\right)\right|^{2}\right) \leqq C_{1}(r+\epsilon)^{2 n} /\left(1-(r+\epsilon)^{2}\right) .
$$

Let $r<r^{\prime \prime}<r^{\prime}$. The functions $q_{n}^{*}$ are all regular in and on $C_{r^{\prime \prime}}$ and we have by (39),

$$
\begin{aligned}
\left|L_{k}^{*}\left(q_{n}^{*}\right)\right|^{2} \leqq M\left(r^{\prime \prime}, \epsilon\right)\left(\int_{c_{r^{\prime \prime}}}\left|q_{n}^{*}(z)\right|^{2} d s\right)\left(\frac{1}{r^{\prime \prime}}+\epsilon\right)^{2 k} \\
\quad(k=0,1, \cdots ; n=0,1, \cdots) .
\end{aligned}
$$

Since the $q_{n}^{*}$ are orthonormal over the interior of $C_{r^{\prime}}$, we have $q_{n}^{*}(z)=o(1)$ uniformly on any closed subset in $C_{r^{\prime}}$. Thus, $\int_{C_{r^{\prime \prime}}}\left|q_{n}^{*}(z)\right|^{2} d s=o(1)(n \rightarrow \infty)$ and $\sum_{k=n}^{\infty}\left|L_{k}^{*}\left(q_{n}^{*}\right)\right|^{2} \leqq M_{1}\left(r^{\prime \prime}, \epsilon\right)\left(1 / r^{\prime \prime}+\epsilon\right)^{2 n}$. The convergence of (43) now follows.

Suppose, conversely, that $T$ is bounded over $L^{2}\left(C_{r^{\prime \prime}}\right), 0<r^{\prime \prime}<r<1$. We shall show that $\lim \sup _{n \rightarrow \infty}\left|T\left(\phi_{n}^{*}\right)\right|^{1 / n} \leqq r$. Any sum $\sum_{n=0}^{\infty} a_{n} \phi_{n}^{*}(z)$ with $\lim \sup _{n \rightarrow \infty}\left|a_{n}\right|^{1 / n} \leqq 1 / r$ is a regular function $f(z)$ in the interior of $C_{r}$, and hence is of class $L^{2}\left(C_{r^{\prime \prime}}\right)$. The convergence of the series is uniform in any closed subset interior to $C_{r}$. Thus, $T(f)=\sum_{n=0}^{\infty} a_{n} T\left(\phi_{n}^{*}\right)$ is convergent for all $a_{n}$ with $\lim \sup _{n \rightarrow \infty}\left|a_{n}\right|^{1 / n} \leqq 1 / r$, and, in particular, for $a_{n}=1 / r^{n}$. Thus, $\left|T\left(\phi_{n}{ }^{*}\right) r^{-n}\right| \leqq M(n=0,1, \cdots)$. The desired inequality now follows. The equality signs in (40) may be proved under the hypothesis of the theorem in the usual way.

In our last theorems, we shall relate the continuability properties of the Riesz representative $l(z)$ of a functional $L$ with the extendability properties of $L$ as an operator. In what follows our discussion is limited to regions with analytic boundaries. We first prove the following result.

THEOREM. Let $B$ be a simply-connected region bounded by an analytic Jordan curve. Let $l(z)$ be regular in a region $B^{+}$for which $B^{-} \subset B^{+}$. Then the function

$$
c(z)=\iint_{B} \frac{[l(w)]^{-}}{z-w} d A_{w}
$$

may be continued analytically from the exterior of $B$ across the boundary of $B$ to the exterior of a suitably chosen region $G$ with $G-\subset B$.

Proof. We introduce the set (a) of functionals $L_{n}(f)=f^{(n)}(0)(n=0,1, \cdots)$, their Riesz representatives $\phi_{n}(z)$, the orthogonalized $\phi$ 's which will be designated by $\phi_{n}^{*}$, and the related biorthogonal set $L_{n}^{*}(f)=\sum_{k=0}^{n} a_{n k} f^{(k)}(0)$. For $f \in L^{2}(B)$, the functionals $L_{n}^{*}$ have the alternate representation $L_{n}^{*}(f)$ $=\iint_{B} f\left[\phi_{n}^{*}\right]-d A$. For a simply-connected domain, it may be shown that

$$
\phi_{n}^{*}(z)=\left(\frac{n+1}{\pi}\right)^{1 / 2} h^{\prime}(z)[h(z)]^{n} \quad(n=0,1, \cdots)
$$


where $h(z), h(0)=0, h^{\prime}(0)>0$, maps the interior of $B$ onto the interior of the unit circle. The functions $\phi_{n}{ }^{*}$ are orthogonal over the interior of every kreisbild, and indeed,

$$
\iint_{C_{r}} \phi_{n}^{*}\left[\phi_{m}^{*}\right]-d A=\frac{((m+1)(n+1))^{1 / 2}}{\pi} \iint_{|t|<r} t^{n}\left\lfloor^{m}\right\rfloor-d A_{t}=r^{2 n+2} \delta_{m n} .
$$

Let us consider now $1 /(z-w)$ as a function of $w$. For $z$ exterior to $B$, $1 /(z-w)$ is regular throughout $B$. For $z$ interior to $B$, there will be a kreisbild $C_{r(z)}$ which passes through $z$ and such that $1 /(z-w)$ is regular in the interior of $C_{r(z)}$. If we set $r(z)=1$ for $z$ exterior to $B$, then we may write

$$
\frac{1}{z-w}=\sum_{n=0}^{\infty} L_{n, w}^{*}\left(\frac{1}{z-w}\right) \phi_{n}^{*}(w)
$$

converging uniformly and absolutely on any closed set interior to $C_{r(z)}$. We also have

$$
\begin{aligned}
L_{n, w}^{*}\left(\frac{1}{z-w}\right) & =\left.\sum_{k=0}^{n} a_{n k} \frac{\partial^{k}}{\partial w^{k}}\left(\frac{1}{z-w}\right)\right|_{w=0} \\
& =\sum_{k=0}^{n} \frac{a_{n k} k !}{z^{k+1}} \equiv \frac{1}{z} P_{n}\left(\frac{1}{z}\right)
\end{aligned}
$$

where

$$
P_{n}(t)=\sum_{k=0}^{n} a_{n k} k ! t^{k} \quad(n=0,1, \cdots)
$$

are a set of polynomials of Faber type for the domain $B$. We have therefore

$$
\frac{1}{z-w}=\sum_{n=0}^{\infty} \frac{1}{z} P_{n}\left(\frac{1}{z}\right) \phi_{n}^{*}(w) \text {. }
$$

If now $r^{-}(z)$ designates any quantity such that $0<r^{-}(z)<r(z)$, then by (48) we have

$$
\iint_{C_{r^{-}(z)}}\left|\frac{1}{z-w}\right|^{2} d A_{w}=\sum_{n=0}^{\infty}\left|\frac{1}{z} P_{n}\left(\frac{1}{z}\right)\right|^{2} \cdot\left(r^{-}(z)\right)^{2 n+2} .
$$

From (53), there is obtained the estimate

$$
\left|\frac{1}{z} P_{n}\left(\frac{1}{z}\right)\right| \leqq \frac{1}{\left(r^{-}(z)\right)^{n+1}}\left[\iint_{C_{r}-(z)} \frac{d A_{w}}{|z-w|^{2}}\right]^{1 / 2} .
$$

We consider now the integral (46). For $z$ exterior to $B$, the series (52) converges uniformly and absolutely for $w \in B$, hence we may write 


$$
\begin{aligned}
c(z) & =\iint_{B}[l(w)]-\left[\sum_{n=0}^{\infty} \frac{1}{z} P_{n}\left(\frac{1}{z}\right) \phi_{n}^{*}(w)\right] d A_{w} \\
& =\sum_{n=0}^{\infty} \frac{1}{z} P_{n}\left(\frac{1}{z}\right) \iint_{B}[l(w)]-\phi_{n}^{*}(w) d A_{w} \\
& =\sum_{n=0}^{\infty}\left[L_{n}^{*}(l(w))\right]^{-} \frac{1}{z} P_{n}\left(\frac{1}{z}\right)
\end{aligned}
$$

valid for $z$ exterior to $B$. We shall show that the series (55) converges uniformly in a region $R$ which includes the exterior of $B$ plus a small strip interior to $B$ adjacent to and including the boundary of $B$. In this way, the series (55) will yield an analytic continuation of $c(z)$ across the boundary of $B$. Since the boundary of $B$ is analytic, the mapping function $h(z)$ will be regular and schlicht throughout a $C_{r_{1}}, r_{1}>1$. Hence, we must also have for $1 \leqq r<r_{1}, \iint_{C_{r}} \phi_{n}^{*}\left[\phi_{m}^{*}\right]-d A=r^{2 n+2} \delta_{m n}$, and the set $\left\{\phi_{n}^{*} / r^{n+1}\right\}$ is orthonormal over $C_{r}$. By hypothesis $l(z)$ is regular in a larger region $B^{+}$and will a fortiori be of class $L^{2}\left(C_{r_{2}}\right)$ for some $1<r_{2}<r_{1}$. We can therefore write

$$
l(w)=\sum_{n=0}^{\infty} \beta_{n} \phi_{n}^{*}(w) / r_{2}^{n+1}
$$

with $\sum_{n=0}^{\infty}\left|\beta_{n}\right|^{2}<\infty$, convergent uniformly interior to $C_{r_{2}}$, and, therefore, $\iint_{B}[l(w)]^{-} \phi_{n}^{*}(w) d A=\beta_{n}^{-} / r_{2}^{n+1}$. We now have

$$
c(z)=\sum_{n=0}^{\infty} \frac{\beta_{n}^{-}}{r_{2}^{n+1}} \frac{1}{z} P_{n}\left(\frac{1}{z}\right) .
$$

Let the quantities $r^{\prime}$ and $r^{\prime \prime}$ satisfy $r_{2}^{-1}<r^{\prime}<r^{\prime \prime}<1$. Then, for all $z$ exterior to $C_{r^{\prime \prime}},(57)$ will be dominated by $(M / \delta)\left(\text { area }\left(C_{r^{\prime}}\right)\right)^{1 / 2} \cdot \sum_{n=0}^{\infty}\left(r^{\prime} r_{2}\right)^{-n-1}<\infty$. Here $M$ is such that $\left|\beta_{n}\right|<M(n=0,1, \cdots)$ and $\delta=\min \operatorname{dist}\left(C_{r^{\prime}}, C_{r^{\prime \prime}}\right)$. The proof is now complete.

THEOREM. Let $L$ be a bounded linear functional over $L^{2}(B)$ where $B$ is a simply-connected region with an analytic boundary. The Riesz representative $l(z)$ of $L$ can be continued analytically to some $B^{+}, B^{-} C B^{+}$if and only if $L$ can be extended so as to be bounded over $L^{2}\left(G^{-}\right), G^{-} \subset B$.

Proof. Suppose that $L$ is continuable to $L^{2}\left(G^{-}\right)$. Then as we have seen above, $L$ possesses a Cauchy representative $c(z)$, and by (29), its Riesz representative is given by $[l(z)]^{-}=\int_{C} c(w) K_{B}\left(z^{-}, w\right) d w$. If $w$ is confined to a closed bounded set interior to $B$, then it is easily shown that if the boundary of $B$ is analytic, $\left(K_{B}\left(z^{-}, w\right)\right)^{-}$will be an analytic function of $z$ in some region $B^{+}: B^{-} C B^{+}$. It follows then that $l(z)$ must be regular in $B^{+}$. Conversely, let $l(z)$ be regular in a $B^{+}$and introduce $c(z)$ by means of (30). By the previous theorem, $c(z)$ will be regular in the exterior of a region $G^{*}$ with $G^{*-} \subset B$. By 
remarks made at the beginning of the section $c(z)$ must be the Cauchy representative of $L$. It follows then that $L$ can be extended to some $L^{2}\left(G^{-}\right)$with $G^{-} \subset B$.

\section{BIBLIOGRAPHY}

1. N. Aronszajn, Theory of reproducing kernels, Trans. Amer. Math. Soc. vol. 68 (1950) pp. 337-404.

2. S. Bergman, The kernel function and conformal mapping, Mathematical Surveys, vol. 5, New York, American Mathematical Society, 1950.

3. T. Carleman, Über die Approximation analytischer Funktionen durch lineare Aggregate von vorgegebenen Potenzen, Arkiv för Matematik, Astronomi och Fysik vol. 17 (1923).

4. P. Davis, On the applicability of linear differential operators of infinite order to functions of class $L^{2}(B)$, Amer. J. Math. vol. 74 (1952) pp. 475-491.

5. P. Davis and H. Pollak, Linear functionals and analytic continuation problems, Pacific Journal of Mathematics vol. 3 (1953) pp. 47-72.

6. P. P. Korovkin, The asymptotic representation of polynomials orthogonal over a region, Doklady Akad. Nauk SSSR. N.S. vol. 58 (1947) pp. 1883-1885.

7. S. Pincherle, Funktionalgleichungen und Operationen, Encyklopaedie der Mathematischen Wissenschaften, vol. 2/1, Heft 6.

8. J. L. Walsh, Interpolation and approximation, Amer. Math. Soc. Colloquium Publications, vol. 20, New York, 1935.

9. J. L. Walsh and P. Davis, Interpolation and orthonormal systems, Journal d'Analyse Mathématique vol. 2 (1952) pp. 1-28.

10. J. M. Whittaker, Séries de base de polynomes quelconques, Paris, 1949.

11. D. V. Widder, A generalization of Taylor's series, Trans. Amer. Math. Soc. vol. 30 (1928) pp. 126-154.

American University,

Washington, D. C.

HARVARD UNIVERSITY,

Cambridge, Mass. 